\title{
Independent perturbations for physics parametrization tendencies in a convection-permitting ensemble (pSPPT)
}

\author{
Clemens Wastl, Yong Wang, Aitor Atencia, and Christoph Wittmann \\ Department of Forecasting Models, Zentralanstalt für Meteorologie und Geodynamik, Vienna, Austria
}

Correspondence: Yong Wang (yong.wang@zamg.ac.at)

Received: 19 July 2018 - Discussion started: 24 September 2018

Revised: 26 November 2018 - Accepted: 3 January 2019 - Published: 16 January 2019

\begin{abstract}
A modification of the widely used SPPT (Stochastically Perturbed Parametrisation Tendencies) scheme is proposed and tested in a Convection-permitting - Limited Area Ensemble Forecasting system (C-LAEF) developed at ZAMG (Zentralanstalt für Meteorologie und Geodynamik). The tendencies from four physical parametrization schemes are perturbed: radiation, shallow convection, turbulence, and microphysics. Whereas in SPPT the total model tendencies are perturbed, in the present approach (pSPPT hereinafter) the partial tendencies of the physics parametrization schemes are sequentially perturbed. Thus, in PSPPT an interaction between the uncertainties of the different physics parametrization schemes is sustained and a more physically consistent relationship between the processes is kept. Two configurations of pSPPT are evaluated over two separate months (one in summer and another in winter). Both schemes increase the stability of the model and lead to statistically significant improvements in the probabilistic performance compared to a reference run without stochastic physics. An evaluation of selected test cases shows that the positive effect of stochastic physics is much more pronounced on days with high convective activity. Small discrepancies in the humidity analysis can be dedicated to the use of a very simple supersaturation adjustment. This and other adjustments are discussed to provide some suggestions for future investigations.
\end{abstract}

\section{Introduction}

Stochastic physics schemes are used worldwide in many ensemble prediction systems (EPSs) to represent uncertainties related to simplifications and approximations in the numerical model itself. Such uncertainties are defined as "model er- ror" and arise from different sources such as computational constraints, incomplete knowledge of physical processes, uncertain parameters in parametrizations, and from discretization methods. These errors range from large spatial scales (e.g., use of climatological aerosol fields) to very small scales due to the use of parametrizations of unresolved processes such as the microphysics or turbulence scheme.

Stochastic parametrization schemes produce an ensemble of perturbed members where each member sees a different, but equally likely, stochastic forcing. They have been shown to significantly improve the reliability of weather forecasts (Sanchez et al., 2016; Leutbecher et al., 2017). Process-based stochastic approaches address sources of uncertainty in a particular parametrization scheme (Plant and Craig, 2008; Bengtsson et al., 2013; Kober and Craig, 2016), while more general approaches treat uncertainty from a number of processes with one single scheme. The most popular method of the latter is the Stochastically Perturbed Parametrisation Tendencies scheme (SPPT) and has been developed at the ECMWF (European Centre for Medium-Range Weather Forecasts; Buizza et al., 1999; Palmer et al., 2009). In SPPT a spectral pattern generator produces random noise with prescribed amplitude and correlations in time and space. This multiplicative noise is used to perturb model tendencies of temperature $(T)$, water vapor content $(Q)$ and wind $(U, V)$. SPPT is operational at forecasting centers worldwide (e.g., ECMWF, UK Met Office, Japan Meteorological Agency, etc.). It has also been proven to work for some limited-area models at the convection-permitting scale, such as AROME (Applications of Research to Operations at Mesoscale; Bouttier et al., 2012) or WRF (Weather Research and Forecasting; Berner et al., 2015). SPPT improves the reliability of forecasts by reducing biases in the ensemble forecasts and 
yields a greater ensemble spread (Weisheimer et al., 2014; Leutbecher et al., 2017).

An often-mentioned shortcoming of the SPPT approach is the lack of physical consistency (Ollinaho et al., 2017; Leutbecher et al., 2017). SPPT only perturbs the net physics tendencies inducing an inconsistency with fluxes computed from unperturbed tendencies (e.g., surface fluxes if surface tendencies are not perturbed). This creates an energy imbalance where individual ensemble members no longer conserve energy. To avoid numerical instabilities based on this misbalance, a tapering function has been introduced to SPPT in the IFS (Integrated Forecasting System) model of ECMWF. It reduces the perturbations smoothly to zero in the boundary layer and in the stratosphere. However, this tapering function destroys the physical consistent representation of model uncertainty in the vertical because it assumes a reduced model error in the lowest and topmost parts of the atmosphere.

Furthermore, the original SPPT generates only a single stochastic pattern, which is applied to the parametrized net tendencies of model variables. This implies that the different schemes are perfectly correlated with each other and have the same error characteristics. This assumption is not always valid as demonstrated by Shutts and Pallares (2014). They have shown, for example, that the uncertainty in the cloud and convection scheme is much higher than in the radiation scheme. Following this discrepancy, Sanchez et al. (2016) have developed a method where a multiplicative noise with different standard deviations for different processes (e.g., gravity-wave drag, boundary layer scheme) is applied to the Unified Model (UM) of the Met Office. Decoupled perturbations among the different schemes increase the ensemble spread, especially in the tropics. However, a tapering function is still needed to ensure numerical stability.

Applying multiplicative noise to net physics tendencies, as in SPPT, implies that the uncertainty representation vanishes where the total tendency is zero. This is also the case if the tendencies from different physics parametrizations are large but act in opposite directions. To overcome this problem, Christensen et al. (2017) have modified the SPPT scheme in the IFS model by perturbing the tendencies of the physics parametrizations with independent stochastic patterns. This perturbation is done at the end of each time step, so no interaction of the uncertainties between the schemes within a time step is considered. This limitation is addressed in the present paper.

In this study, we propose a modified SPPT approach in which the physical consistency between the different parametrization schemes is kept. The details of two different versions of the developed scheme are described in Sect. 2. Section 3 contains a comparison of these schemes with the SPPT approach for two recent test periods (July 2016, January 2017). Standard probabilistic scores are used for surface and upper-air variables. In Sect. 4 the effect of stochastic physics is analyzed on days with strong convection over the Alpine test area and compared to days with stable conditions.
Section 5 contains a summary of the results together with a discussion and the final conclusions.

\section{Experimental design and methodology}

\subsection{The C-LAEF system}

The C-LAEF (Convection-permitting - Limited Area Ensemble Forecasting) system has been developed at the Austrian national meteorological service ZAMG (Zentralanstalt für Meteorologie und Geodynamik) and is based on the convection-permitting AROME model (Seity et al., 2011). AROME is under active development within the international NWP (Numerical Weather Prediction) consortia ALADIN (Aire Limitée Adaptation dynamique Développement InterNational; Termonia et al., 2018), HIRLAM (High Resolution Limited Area Model; Bengtsson et al., 2017) and RC LACE (Regional Cooperation for Limited Area Modelling in Central Europe; Wang et al., 2018). AROME has been operationally used at ZAMG since 2014. The model is run on a domain centered on Austria and covers the Alpine region (Fig. 1). It has a grid spacing of $2.5 \mathrm{~km}, 90$ vertical levels and a time step of $60 \mathrm{~s}$. The nonhydrostatic dynamical kernel of AROME is identical to that developed for the ALADIN model (Bubnová et al., 1995; Bénard et al., 2010). The AROME physics package is mainly adopted from the research model Meso-NH (Mascart and Bougeault, 2011) with the following main components: one moment bulk microphysical scheme ICE3 (using three prognostic ice and hydrometeor classes; Pinty and Jabouille, 1998); statistical sedimentation of falling hydrometeor species after Bouteloup et al. (2011); a 1-D 1.5-order turbulence scheme (Cuxart et al., 2000); a mass-flux-type shallow convection scheme with turbulence closure (Pergaud et al., 2009); no deep convection scheme (because deep convection is assumed to be resolved by the dynamics); and three-layer surface scheme SURFEX (Surface Externalisée; Masson et al., 2013) using a tile approach including sub-schemes for land, vegetation, town, sea, and lake. The radiation scheme for AROME is taken from the ECMWF IFS model where short-wave radiation is computed after Fouquart and Bonnel (1980) and long-wave using the Rapid Radiative Transfer Model (RRTM; Mlawer et al., 1997).

The C-LAEF ensemble comprises 16 members using the first 16 out of a total of 51 members of ECMWF-ENS (ensemble system of the ECMWF IFS model) for the boundary conditions. Coupling is done every $3 \mathrm{~h}$ using a Davies relaxation scheme (Davies, 1976). Weidle et al. (2013) have shown that 16 members are a good compromise between ensemble size and computational costs. The ECMWF-ENS global ensemble system is operated on a cubic octahedral grid with about $0.2^{\circ}$ horizontal resolution and 91 vertical levels. The members are created via a combination of ensemble data assimilation (Isaksen et al., 2010) and singular vectors 


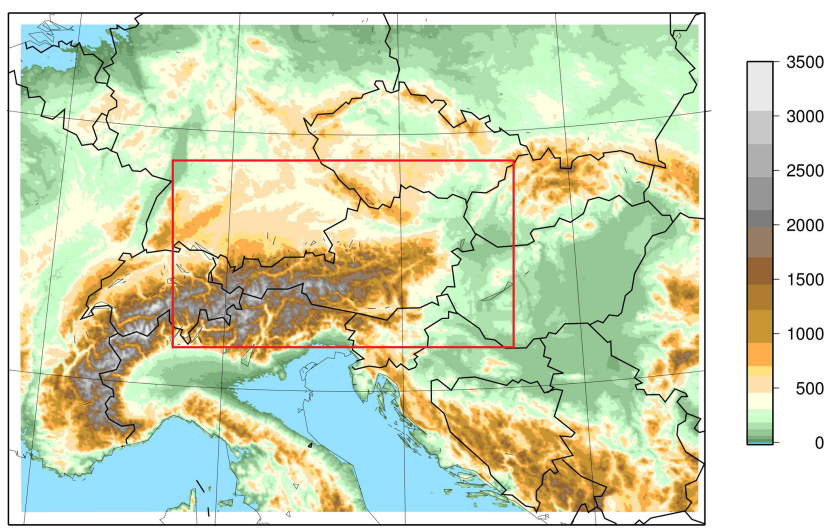

Figure 1. Domain of the C-LAEF system including the INCA domain for precipitation verification (red). The coloring shows the altitude (m).

(Leutbecher and Lang, 2013) for the initial state and by using SPPT and the stochastic kinetic energy backscatter (SKEB) method (Berner et al., 2009) during model integration.

Since the authors are only interested in the effect of stochastic physics, no extra initial or boundary condition perturbations are applied on the C-LAEF side. For the same reason, no data assimilation is used in the experiments and surface uncertainty is not taken into account either. These assumptions are deemed acceptable because only the difference between stochastic physics perturbation schemes are studied. The C-LAEF system is run once per day (00:00 UTC) with a forecast range of $30 \mathrm{~h}$ and an output frequency of $1 \mathrm{~h}$.

\subsection{Stochastic physics schemes}

\subsubsection{SPPT}

The original SPPT stochastic physics scheme was initially developed by Buizza et al. (1999) for the IFS model of the ECMWF. Palmer et al. (2009) modified the scheme by introducing a spectral pattern generator. It creates a random 2-D field with a prescribed standard deviation and temporal and spatial correlation length. In the IFS implementation, three independent random patterns with different correlation scales are used. They are designed to span the uncertainty at mesoscale, synoptic scale, and planetary time and space scales. The resulting random patterns are Gaussian distributed with zero mean, unit variance, and a homogeneous and isotropic horizontal autocorrelation. The amplitude of the perturbations is restricted to a range defined by the standard deviation $[-2 \sigma, 2 \sigma]$. The net tendencies, $P$, of wind ( $U$ and $V$ component), temperature ( $T$ ), and water vapor content $(Q)$ are multiplied at each time step during the model integration with this perturbation field to generate the perturbed physics tendencies. The perturbed net tendency of the physics parametrizations $\left(P^{\prime}\right)$ at each grid point is repre-
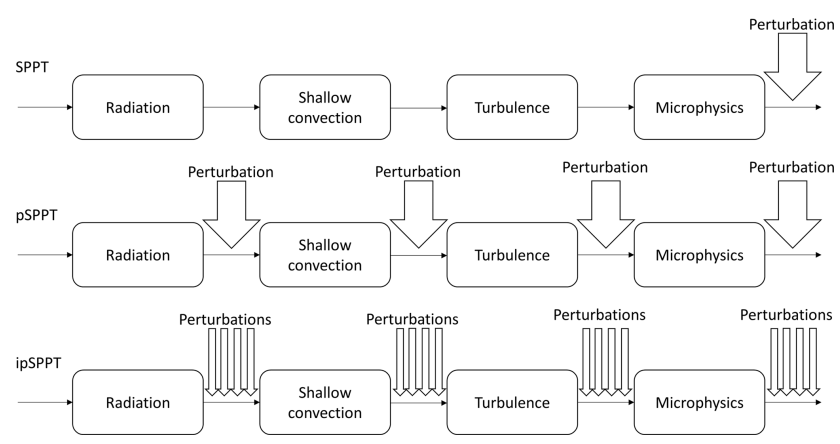

Figure 2. Illustration of how the stochastic perturbations are applied in the different physics parametrization schemes of SPPT (first row), pSPPT (second row), and ipSPPT (last row).

sented by:

$P^{\prime}=(1+\alpha r) \sum_{i=1}^{n} P_{i}$,

where $\alpha$ is a level dependent constant defined by a tapering function, $r$ is a random number defined by the perturbation pattern, $P_{i}$ is the unperturbed tendency of one parametrization scheme, and $n$ is the number of physics schemes contributing to the total tendency equation. The first row in Fig. 2 illustrates how the physics tendencies of C-LAEF are perturbed in SPPT. Due to the multiplicative feature, the scheme attributes the greatest uncertainties to the areas where the largest net tendencies $P$ occur. The shape of the tapering function $\alpha$ can be controlled in the model setup. It reduces the perturbations to zero in the boundary layer below $900 \mathrm{hPa}$ (default) and in the stratosphere above $100 \mathrm{hPa}$ (default). $\alpha$ is set to 1 for all remaining levels, thereby retaining the vertical structure that results from the physics parametrizations. The tapering function has been introduced to the IFS model to avoid numerical instabilities - it is not necessary in some regional models like WRF or COSMO (Leutbecher et al., 2017).

Bouttier et al. (2012) have successfully implemented SPPT in the AROME model. Some changes have to be made to the original SPPT in order to adapt the methodology from IFS to AROME. The main change is the adaption of the spectral pattern generator from the spherical harmonics applied in the IFS to the bi-Fourier functions used in AROME. The link between the variance spectrum and the bi-Fourier representation follows the formulation by Berre (2000). At the edges of the model domain, the uncertainties originate only from the lateral boundary formulation and the physical tendencies are smoothly relaxed to zero. Due to the relatively short forecast range of the convection-permitting AROME model $(30 \mathrm{~h})$, only one stochastic pattern is used instead of three in the case of the IFS model. In the AROME implementation of SPPT, no perturbations of temperature and humidity are applied if the resulting humidity value is negative or exceeds the critical saturation value (supersaturation adjustment; Bouttier et al., 2012). This is different from the IFS version, where a smooth 
humidity reduction is applied in such cases (Palmer et al., 2009). The default settings of the pattern generator applied by Bouttier et al. (2012) have to be tuned to the C-LAEF configuration. Using SPPT in the AROME model requires a tapering function to avoid numerical instabilities. Experiments with tapering off in the boundary layer in SPPT resulted in several model crashes during the test period because of too strong wind over the Alps. However, this has not been further investigated. The main characteristic of this scheme, described as "SPPT" hereinafter, is the perturbation of net tendencies without considering the contribution of each individual physics tendencies (Fig. 2). In other words, this approach assumes that no uncertainty is added when the net tendency is zero, even though the single physics schemes might have large but compensating contributions.

\subsubsection{Physical parametrization-based SPPT (pSPPT)}

The restrictions and assumptions made in the original SPPT approach have led to the idea of setting up a modified version of SPPT. The main goal is to maintain the interactions between the individual physics schemes, and thus, to keep the model stable. The different physics schemes in AROME are called in the following order: radiation, shallow convection, turbulence, and microphysics. Each scheme provides a partial tendency of the main model quantities $T, U, V$, and $Q$. The condensed water species are not directly perturbed, they are adjusted at each time step by the fast microphysics step (Seity et al., 2011). In the original SPPT version the partial tendencies of the different physics parametrizations are summed up at the end of the time step and this net tendency is finally perturbed by the noise of the pattern generator as in Eq. (1). As a consequence, the uncertainties resulting from one scheme are not passed to the following scheme.

In the present study, it is proposed to perturb the partial tendencies of the physics schemes separately and to consider the resulting perturbed fields in the subsequent physics scheme. We call this approach physical parametrizationbased SPPT (pSPPT hereinafter). Equation (2) shows the formulation of the perturbed partial tendency of each parametrization scheme in this new pSPPT scheme; an illustration of this is given in Fig. 2. Each random pattern $\left(r_{i}\right)$ is generated separately by the pattern generator using a different seed.

$P_{i}^{\prime}=\left(1+\alpha r_{i}\right) P_{i}$ for $i=1, n$

The uncertainties are passed through the different schemes and as a consequence the issue of only perturbing nonzero net tendencies is avoided. For example, if the turbulence scheme provides a strong positive temperature tendency and the microphysics scheme a comparable negative temperature tendency, no effect of stochastic physics perturbations is present in the original SPPT. However, pSPPT will either intensify or weaken the strong positive tendency of the turbulence scheme, depending on the stochastic pattern. The resulting tendency is then processed in the microphysics scheme and afterwards again adapted by the perturbation process. This approach has a positive effect on the stability of the model, as shown by a reduction of the number of model crashes in a sensitivity study during the 2011 test period. The increased numeric stability in PSPPT allows for the tapering function for microphysics, radiation, and shallow convection schemes to be switched off, being only maintained for the turbulence scheme. In the turbulence scheme, the stochastic perturbations in the lower atmosphere produce too much instability and therefore the model crashes after some time steps. A potential drawback of the pSPPT approach is a possible duplication in attributing errors across schemes, which can introduce inherent correlations between the perturbations applied to one physics scheme and the output of a later scheme (Christensen et al., 2017).

\subsubsection{Independent physical parametrization-based SPPT (ipSPPT)}

In pSPPT as well as in SPPT, the tendencies of all considered variables $(T, U, V$, and $Q)$ are perturbed with the same stochastic pattern, which assumes that the different variables in the parametrization schemes have similar error characteristics. However, this assumption is vague and might not always be satisfied as Boisserie et al. (2013) have shown. This leads us to a new approach where the tendencies resulting from the physical parametrization schemes (temperature, wind components, and water vapor content) are perturbed by individual stochastic patterns. It can be seen as an adaptation of the pSPPT approach presented before and is called ipSPPT hereinafter. Equation (3) highlights the independence of this ipSPPT methodology, by formulating the perturbation of $T$, $U, V$, and $Q$ separately. An illustration of this is given in the last row of Fig. 2.

$$
\begin{aligned}
T_{i}^{\prime} & =\left(1+\alpha r_{i, 1}\right) T_{i} ; U_{i}^{\prime}=\left(1+\alpha r_{i, 2}\right) U_{i} ; V_{i}^{\prime} \\
& =\ldots \text { for } i=1, n
\end{aligned}
$$

As a consequence, the random field applied to, for example, the temperature tendency $(T)$ is different from the one used for the wind components $(U, V)$ or the water vapor content $(Q)$. Tapering is treated in ipSPPT as in the pSPPT approach (active only for the turbulence scheme). The first SPPT version in the IFS model (Buizza et al., 1999) has also used such separate patterns for the different parametrized tendencies. However, it has been removed in the revised SPPT scheme (Palmer et al., 2009) because some physical relationships within a parametrization scheme could be violated in this way (see Sect. 5).

\subsection{Experimental setup and verification methods}

A 2-week period (16-30 July 2011) is used to optimize the settings of the spectral pattern generator and the different parameters of the stochastic physics schemes in the C-LAEF 
system. The goal of this optimization is to generate a realistic spread without creating a model bias. A set of four experiments has been chosen for a long-period verification: one experiment without any stochastic physics perturbations (REF), one containing the original SPPT approach (SPPT Sect. 2.2.1), a version using physical parametrization-based SPPT (pSPPT - Sect. 2.2.2), and a version of pSPPT with independent patterns for the prognostic variables (ipSPPT Sect. 2.2.3). The experimentation is conducted over a summer month (July 2016) and winter month (January 2017) with one run per day (00:00 UTC) and $30 \mathrm{~h}$ forecast range. The model domain is shown in Fig. 1 and corresponds to the operational deterministic AROME domain used at ZAMG.

The upper-air weather variables are verified using ECMWF analyses at the 500 and $850 \mathrm{hPa}$ levels, while surface variables are verified using SYNOP station data. Forecast values are interpolated to the observation location for smooth fields such as $2 \mathrm{~m}$ temperature, $10 \mathrm{~m}$ wind speed, or surface pressure. In the case of precipitation, the forecasts are matched to the nearest grid point. A height correction is applied to the $2 \mathrm{~m}$ temperature to account for discrepancies between model surface and station height. The verification is performed over the whole C-LAEF domain in Fig. 1 which contains more than 1200 observation sites. Beside classical scores such as ensemble spread, ensemble bias or ensemble root-mean-square error (RMSE), the skill of the forecasts is also evaluated by a set of probabilistic scores like the continuous ranked probability score (CRPS; Wilks, 2011) or the Brier score (BS; Hamill and Colucci, 1997). The statistical significance of the score differences between the three experiments and the reference run is defined by using a bootstrapping confidence test. Therefore a block of 3 days is sampled out of the 31-day verification period (both summer and winter) and the time averaged score difference to the reference run is computed. An empirical distribution of all three experiments is constructed by repeating this procedure for 5000 times. The score difference is deemed significant if its sign is not contradicted by more than $10 \%$ of the sample (for more details see Wilks, 2011).

\section{Results}

\subsection{Summer period: July 2016}

\subsubsection{Upper-air verification}

The large-scale synoptic pattern in the first half of July 2016 was characterized by a very deep trough over the British Islands directing an extensive southwesterly flow over the target area of central Europe. This arrangement resulted in a strong advection of warm and moist air masses towards the Alps leading to strong convective activity. Numerous thunderstorms causing local flash floods and even tornadoes were observed during this time. In the second part of July 2016 a very weak pressure gradient was established over central Europe causing some isolated convection with stationary thunderstorms and locally high precipitation amounts.

Figure $3 \mathrm{a}-\mathrm{d}$ shows the performance of the three experiments (SPPT, pSPPT, ipSPPT) as a difference relative to the reference run without any stochastic physics for temperature (Fig. 3a, b) and wind speed (Fig. 3c, d) at $500 \mathrm{hPa}$ (Fig. 3a, c) and $850 \mathrm{hPa}$ (Fig. 3b, d), respectively. The use of stochastic physics should result in an increase in ensemble spread together with an unmodified, or sometimes reduced model error (Leutbecher et al., 2017). Hence, positive differences in spread and negative differences in RMSE are desirable.

Significant differences are represented by filled circles for ensemble spread and by crosses for RMSE in Fig. 3. The ipSPPT experiment (black) shows the highest gain in spread for both temperature and wind speed at both levels. The original SPPT (red) and the pSPPT approach (blue) also exhibit an increase in spread. Focusing on the RMSE (dashed lines), Fig. 3 reveals a small increase in RMSE for temperature at $500 \mathrm{hPa}$ in all three experiments, especially from forecast hour 12 onwards. For both pSPPT and ipSPPT, this temperature increase is even statistically significant. Interestingly, this feature is not present at $850 \mathrm{hPa}$, where the use of stochastic physics leads to a general decrease in RMSE. A slight temperature increase above $800 \mathrm{hPa}$ has already been observed by Bouttier et al. (2012) in the French AROMEEPS experiment, but no explanation was provided. This effect can partly be explained by the very simple supersaturation adjustment, which is used in our experimentation, but this needs to be further investigated over a longer test period. Perturbations are not applied to temperature and water vapor content when the saturation level is exceeded. Hence, a general trend towards a systematic drying of the atmosphere is implied, because more negative perturbations are applied in total. This drying effect was already highlighted by several SPPT studies (Berner et al., 2009; Bouttier et al., 2012). To overcome this shortcoming, Davini et al. (2017) have developed a moisture conservation fix, which was also adapted to the global IFS model by Leutbecher et al. (2017). An improved supersaturation adjustment has also been developed for the AROME model by Szúcs (2016), but it has not yet been implemented in the present experimentation. Szúcs (2016) evaluated this drying effect for the AROMEEPS model during the convective season in 2015. After $24 \mathrm{~h}$ lead time the use of a simple supersaturation adjustment resulted in a negative bias for relative humidity of about $1 \%$ at $700 \mathrm{hPa}$ and about $2 \%$ at $850 \mathrm{hPa}$ and at the surface. In terms of temperature, the simple supersaturation is translated into a slight temperature increase due to the omission of negative temperature perturbations when the supersaturation level is reached. This temperature effect is not present at lower levels, because the reduced humidity at the surface is compensated by stronger evaporation during the day and rapidly decreasing temperatures during the night (Leutbecher et al., 2017). 

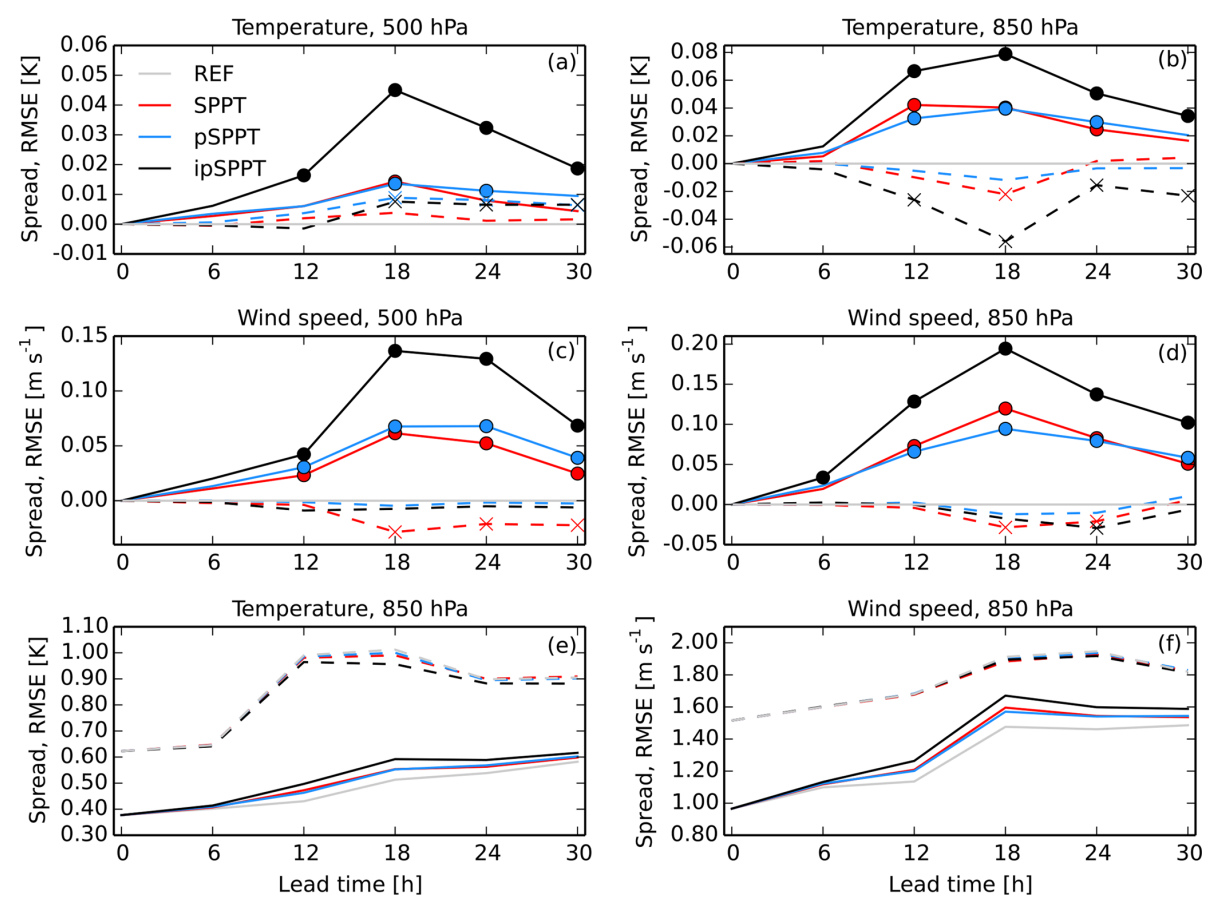

Figure 3. Ensemble spread (solid lines) and RMSE (dashed lines) as a function of lead time for temperature at $500 \mathrm{hPa}$ (a) and $850 \mathrm{hPa}$ (b, e) and wind speed at $500 \mathrm{hPa}$ (c) and $850 \mathrm{hPa}(\mathbf{d}, \mathbf{f})$ in July 2016. Panels (a) to (d) are shown as the differences between an ensemble without any stochastic physics (REF), and circles (crosses) denote significant differences for the ensemble spread (RMSE). Panels (e) and (f) show absolute numbers for all four experiments at $850 \mathrm{hPa}$.

The behavior of the C-LAEF system is indicated by Fig. 3e and $\mathrm{f}$ where the absolute spread and RMSE for temperature and wind speed at $850 \mathrm{hPa}$ is shown. The RMSE is generally high, even at initialization time, because these simulations are pure downscaling of the IFS model without any data assimilation. The spread increases with lead time, while the RMSE is higher during the day when radiation and turbulent fluxes are larger and convection occurs. A spread smaller than the RMSE is an indicator of an underdispersive ensemble. The spread and RMSE lines are closer in the ipSPPT experiment, showing the positive effect of this method on the ensemble performance.

This behavior is also reflected in the probabilistic CRPS (not shown). CRPS measures the skill of the ensemble mean forecast as well as the ability of the perturbations to capture the deviations around it (Bowler et al., 2008). A low value of CRPS indicates a more skillful forecast. For temperature at $850 \mathrm{hPa}$ and wind speed at both 850 and $500 \mathrm{hPa}$, the application of the stochastic physics methods leads to a significant decrease in CRPS, compared to the reference run. Only for temperature at $500 \mathrm{hPa}$ the CRPS difference is slightly positive for all three experiments due to the positive temperature bias. CRPS shows a diurnal cycle similar to RMSE in Fig. 3.

\subsubsection{Surface verification}

The same verification is done for the $2 \mathrm{~m}$ temperature, $10 \mathrm{~m}$ wind speed, mean sea level pressure (MSLP), and precipitation surface variables. Spread and RMSE plots are not shown, but the CRPS is shown in Fig. 4a-d. For temperature and wind speed all three stochastic physics experiments have smaller CRPS values representing a more skillful forecast. This behavior can be explained by an increase in the ensemble spread, while the ensemble average error is not noticeably influenced by the stochastic physics perturbations (not shown). The increase in spread is smallest for the SPPT experiment, which can be attributed to the tapering function in the boundary layer, which is used for all parametrization schemes in this experiment. MSLP in the original SPPT and pSPPT does not show a noticeable impact, but in ipSPPT there is a significant improvement. The ipSPPT results in an improvement in the precipitation verification (reduced CRPS) as well, which is especially significant in the afternoon when convection is abundant during the summer season (Fig. 4). The significant reduction of CRPS for precipitation is mainly caused by a large increase in ensemble spread (not shown).

To investigate the effect of the simple supersaturation treatment in the boundary layer, $2 \mathrm{~m}$ temperature and relative humidity biases relative to the REF experiment are given in Fig. $4 \mathrm{e}$ and $\mathrm{f}$. It reveals a general trend towards lower tem- 

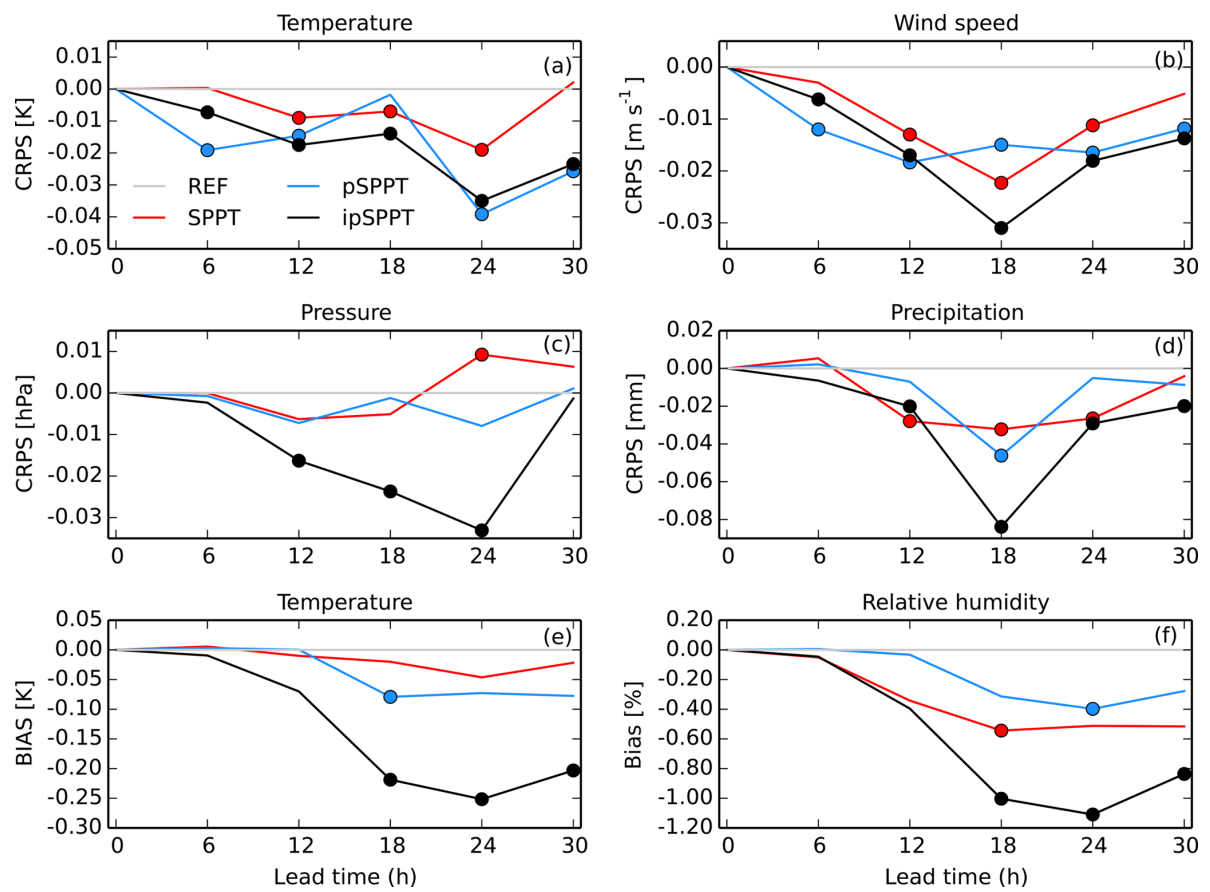

Figure 4. Continuous ranked probability score (CRPS) as a function of lead time for $2 \mathrm{~m}$ temperature (a), $10 \mathrm{~m}$ wind speed (b), mean sea level pressure (c), and precipitation (d) surface variables in July 2016. Panels (e) and (f) show the bias (BIAS) of $2 \mathrm{~m}$ temperature and relative humidity for the same period. All numbers are shown as a difference between C-LAEF without any stochastic physics (REF). Circles denote significant differences in CRPS and BIAS, respectively.

peratures in all experiments with stochastic physics and the strongest effect for the ipSPPT experiment in the afternoon and evening hours. A significant drying of the boundary layer is obvious in all three experiments with stochastic physics and can be attributed to the simple supersaturation adjustment.

Generally, the differences in the scores analyzed in this section are quite small but significance is reached and they are comparable to other studies of stochastic physics on the convection-permitting scale (e.g., Bouttier et al., 2012; Bowler et al., 2008).

\subsection{Winter period: January 2017}

\subsubsection{Upper-air verification}

January 2017 was the coldest January in the last 30 years in most parts of Austria. The weather situation during the first 2 weeks was characterized by a widespread high-pressure system over the eastern Atlantic Ocean blocking the westerlies and enabling the advection of cold polar air masses from the Arctic Sea towards central Europe. Embedded fronts caused strong snow falls resulting in an region-wide snow cover over central Europe. This situation fueled the local production of cold air near the surface during the long winter nights. In the second part of the month, a high-pressure system over Scandinavia caused easterly winds over the Alps advecting extremely cold, continental air masses from Russia into the target domain.

Compared to the summer period verification, the score differences in upper-air variables of January 2017 in Fig. 5 are much smaller. For temperature and wind speed at both levels $(500$ and $850 \mathrm{hPa})$ the use of stochastic physics results in an increase in ensemble spread. However, statistical significance over the whole forecasting range is only reached for temperature and wind speed at $850 \mathrm{hPa}$ in the ipSPPT approach. RMSE is not influenced significantly, except for the wind speed at $850 \mathrm{hPa}$ in the case of ipSPPT. However, a small trend towards higher temperatures and lower humidity in the experiments with stochastic physics also persists in winter (not shown). The CRPS in upper air is slightly decreased for all variables considered in January 2017, but being statistically significant only in the case of ipSPPT (not shown). It seems that the different error representations of the model variables $T, U, V$, and $Q$ have a positive effect on the scores at these levels in winter.

\subsubsection{Surface verification}

The RMSE of the surface variables in C-LAEF is very large for January 2017 (Fig. 6e, f). The bias is strongly positive, especially for $2 \mathrm{~m}$ temperature, indicating significantly higher temperatures in the model than observed. This can be partly explained by the fact that data assimilation is not 

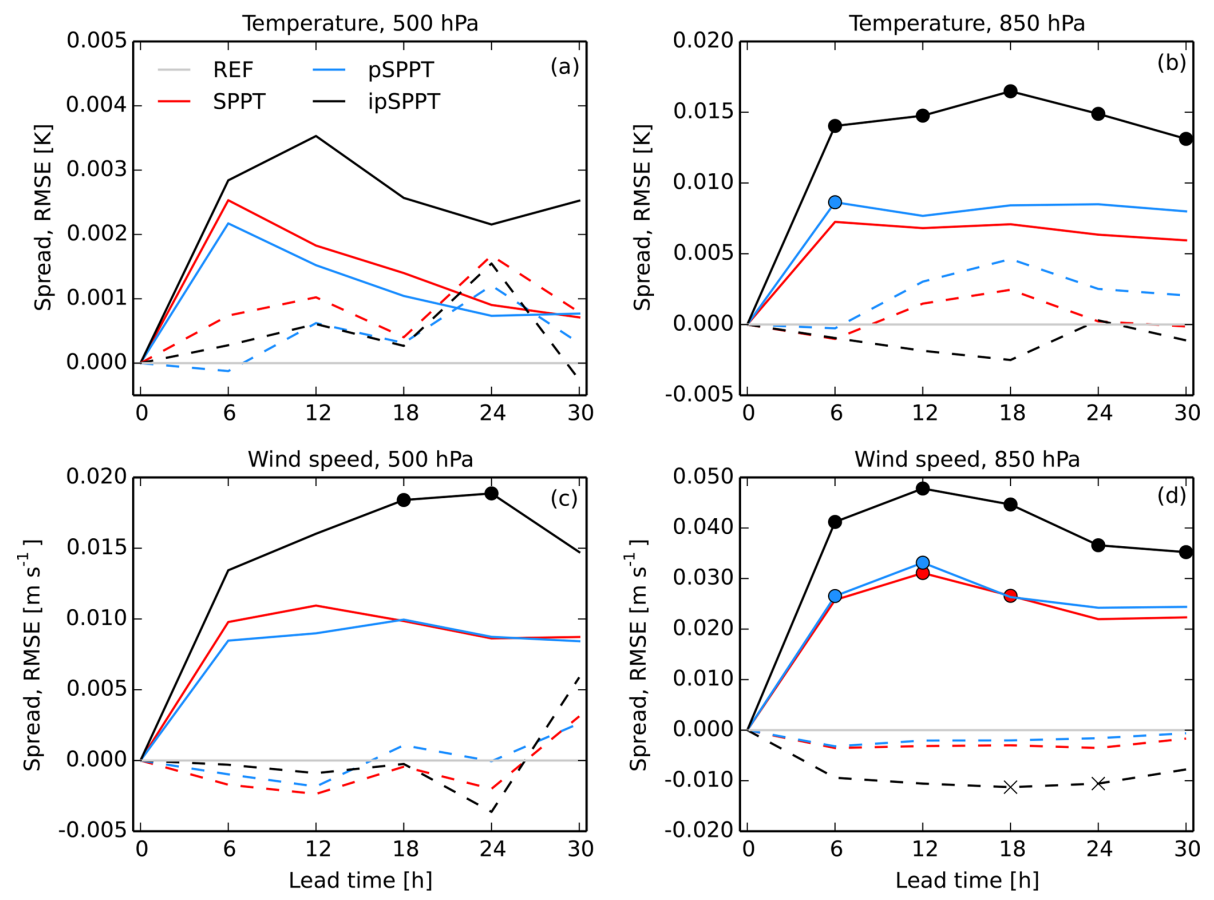

Figure 5. Ensemble spread (solid lines) and RMSE (dashed lines) as a function of lead time for temperature at $500 \mathrm{hPa}$ (a) and $850 \mathrm{hPa}$ (b) and wind speed at $500 \mathrm{hPa}$ (c) and $850 \mathrm{hPa}$ (d) in January 2017. Scores are shown as the differences between an ensemble without any stochastic physics (REF), and circles (crosses) denote significant differences for the ensemble spread (RMSE).

used. However, other operational models at ZAMG also performed poorly during this period, with the pronounced temperature inversions in Alpine valleys posing big problems for the models. C-LAEF simulated a breakup of the temperature inversion in the afternoon, but in reality the cold air was very persistent.

The ensemble spread is much smaller than the model error showing a highly underdispersive ensemble. This fact can be explained by the absence of initial conditions and surface perturbations in our experimentation. Focusing on the improvements compared to the reference ensemble, Fig. 6a-d shows an increase in ensemble spread for the ipSPPT and especially pSPPT experiment, while the original SPPT method does not have a strong effect. This can be attributed to the stronger tapering in SPPT. The pSPPT also produces a significant increase in the RMSE for temperature around noon $(+12 \mathrm{~h})$. Finally, the effect of the simple supersaturation adjustment, which influences the scores in the summer period, is not visible at the surface in January 2017. This is because January 2017 was a rather dry month, with a lot of sunny days where saturation was rarely reached in the lower atmosphere.

The $10 \mathrm{~m}$ wind speed exhibits an increase in spread for all three experiments, while the ensemble average error is barely modified. In the ipSPPT experiment, the RMSE of the MSLP is significantly decreased, which is also reflected in a reduction of CRPS (not shown). The other two experiments instead reveal a RMSE increase compared to REF. For precipitation, the ensemble spread is significantly increased in the ipSPPT experiment and to a lesser extent in the pSPPT scheme. The RMSE of precipitation is decreased for all three experiments between 12 and $24 \mathrm{~h}$ lead time compared to REF.

\section{Impact on convection}

Forecasting convection in summer still remains one of the biggest challenges for the current high-resolution NWP systems, especially in complex terrain like the Alps. Section 3 showed that PSPPT and especially ipSPPT can significantly improve the ensemble spread of precipitation forecasts in summer. To further investigate this behavior, several test cases with high convective activity are selected out of the July 2016 period and compared to days with stable conditions. The selection of cases is based on the Convective Available Potential Energy (CAPE) and the observed precipitation gained from the operational analysis system INCA (Integrated Nowcasting through Comprehensive Analysis; Haiden et al., 2011; Wang et al., 2017). All days with CAPE $>1000 \mathrm{~J} \mathrm{~kg}^{-1}$ in the afternoon (15:00 UTC) averaged over the whole INCA domain (Fig. 1) and some observed thunderstorms are grouped into the convective class, days with CAPE $<500 \mathrm{~J} \mathrm{~kg}^{-1}$ remain in the nonconvective class. Following this classification, 13 days of July 2016 can be assigned to the convective class and 10 days to the nonconvective class. 

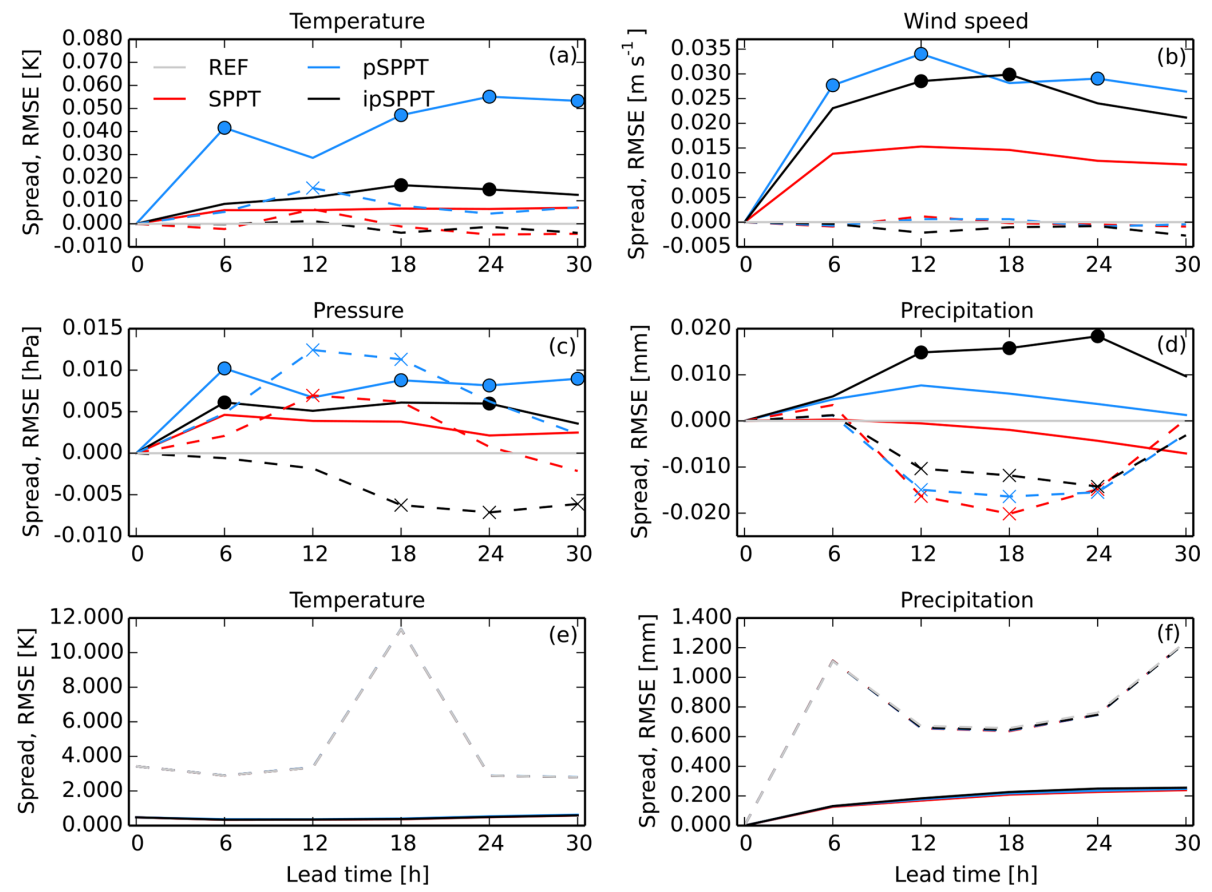

Figure 6. As in Fig. 3, but for $2 \mathrm{~m}$ temperature (a), $10 \mathrm{~m}$ wind speed (b), mean sea level pressure (c), and precipitation (d) surface variables in January 2017. The last row shows absolute numbers for temperature (e) and precipitation (f).

Figure 7 shows the ensemble spread and RMSE for precipitation of all experiments relative to an ensemble without stochastic physics (REF). For this precipitation verification the observations are taken from the INCA analysis system which combines rain gauge and radar data on a $1 \mathrm{~km}$ grid. Comparing the two columns of Fig. 7 reveals a much stronger impact of stochastic physics on the ensemble spread at days with significant convection. Especially for the ipSPPT approach the spread increase (compared to REF) in the afternoon of convective days is about 5 times higher than for days with stable conditions. Also for SPPT and pSPPT the spread increase is mainly restricted to days with convection. The effect on RMSE of precipitation is generally smaller (see also Sect. 3.1.2). A slight reduction of RMSE in the afternoon can be seen for SPPT and PSPPT with the larger values on convective days. The effect on RMSE for the ipSPPT experiment is generally small in both cases. This case study shows that introducing perturbations into a model is much more effective when convection and vertical motion in the atmosphere is high. This is only shown for precipitation in Fig. 7, but also for temperature or wind speed the effect of stochastic physics is much higher at convective days (not shown). This explains why the scores presented in Sect. 3 are generally smaller in winter when the conditions in the considered area are generally much more stable than in summer.

\section{Discussion and conclusions}

In this study we have proposed two physical parametrizationbased SPPT versions (pSPPT, ipSPPT) and have investigated their performance in a convection-permitting ensemble for one summer and one winter month. In pSPPT the partial tendencies of turbulence, radiation, shallow convection, and microphysics are perturbed individually and interact with the subsequent parametrization schemes. In other words, each parametrization sees the updated state including the perturbed tendencies of the previous parametrizations (Fig. 2). In ipSPPT an independent perturbation is additionally applied to the parametrization tendencies of $T, U, V$ and $Q$. These two schemes have been compared to the original SPPT method (Buizza et al., 1999; Bouttier et al., 2012) and a control ensemble without any stochastic perturbations. As expected, the use of stochastic physics increases the ensemble spread, especially in periods with high convective activity (summer period). The gain of spread is clear in temperature and wind speed at all model levels, with the highest increase near the surface. This can be mainly attributed to the reduced tapering of perturbations in the boundary layer in PSPPT and ipSPPT. In the case of precipitation, SPPT has little effect on the ensemble spread, whereas the new ipSPPT scheme reveals a statistically significant increase in ensemble spread compared to the reference experiment. The model error has been analyzed by calculating the RMSE of each experiment as difference to the reference run. For most variables stochastic physics lead to a slight decrease in model error throughout 

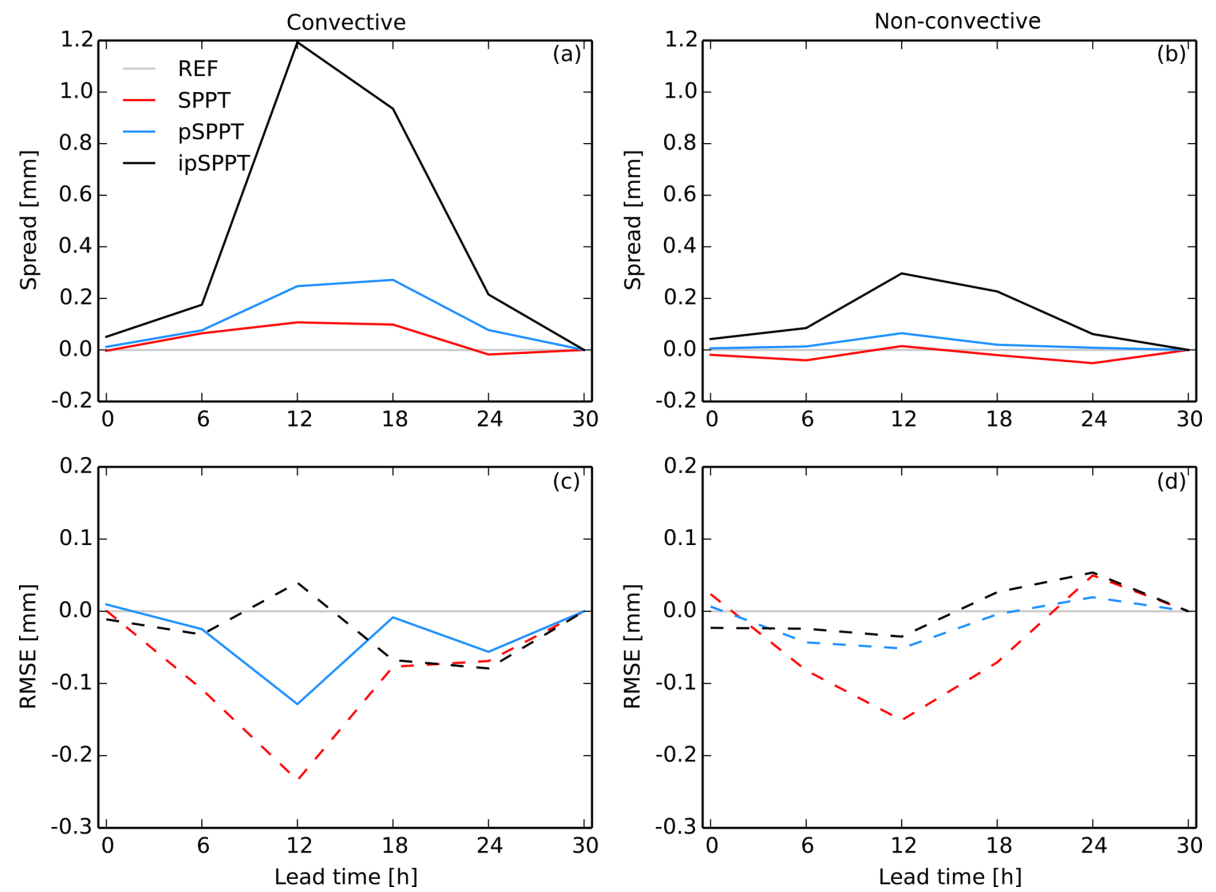

Figure 7. Ensemble spread (a, b) and RMSE (c, d) of precipitation as a function of lead time. The panels (a) and (c) refer to days with high convective activity, the panels (b) and (d) to days with stable conditions. Scores are shown as the differences between an ensemble without any stochastic physics (REF).

all lead times. The strongest effect is observed with the ipSPPT approach. In the case of temperature, the effect is much more complex: a positive temperature bias is observed in the upper levels (e.g., $500 \mathrm{hPa}$ ), while a negative difference of bias is obtained near the surface. The simple supersaturation adjustment used in our experimentation has a strong impact on the temperature and especially humidity scores presented here. This adjustment tends to favor positive temperature and negative water content perturbations due to omitting perturbations when supersaturation is reached. This leads to a significant drying of the atmosphere, which results in a cooling effect in the surface boundary layer due to higher evaporation rates during the day and stronger long-wave emission at night. These problems should be reduced by using an improved supersaturation, adjustment which has already been developed for the AROME model (Szúcs, 2016). However, this has not yet been used in the present study, but will be tested in the near future.

CRPS confirmed the better performance of the ensemble when using stochastic physics perturbations. These improvements are generally much smaller in winter than in summer, which can be explained by the more stable stratification of the atmosphere. A small temperature increase is sufficient to trigger convection and to influence wind, humidity, and precipitation fields in summer. This conclusion is supported by a more in-depth analysis of a set of convective events presented in this paper.
The main reason for trying two new approaches of stochastic physics perturbations is because of the restrictions and assumptions made in the original SPPT. The first assumption is the use of a tapering function that has been implemented in SPPT to consider the imbalance between perturbed atmospheric tendencies and the unperturbed surface fluxes and thus to avoid numerical instabilities. On the other hand, smoothly relaxing the perturbations to 0 in the lowermost levels of the atmosphere implies a different error representation in the vertical, which can be considered physically unsatisfactory. Sensitivity studies during the test period of July 2011 with tapering switched off in the SPPT approach showed that about $10 \%$ of model crashes were due to exceptionally high wind speeds over the Alps. Perturbing the physical schemes separately and considering these perturbed fields in the subsequent parametrization (pSPPT) results in a positive effect on the stability of the model. In this case the tapering function has been switched off for microphysics, radiation, and shallow convection without any problems. For the turbulence scheme, the perturbations in the lower atmosphere produce too much instability, especially in the Alps, and therefore the tapering function has to be turned on. Switching off the tapering function separately for the schemes is only possible in the new independent approaches with partial tendencies (pSPPT, ipSPPT). In the case of the original SPPT, the physical schemes cannot be influenced independently.

The main difference between the pSPPT approach presented here and the independent SPPT (iSPPT) method pro- 
posed by Christensen et al. (2017) is the time when the perturbations are applied. In iSPPT the stochastic perturbations are applied at the end of the time step; whereas in the approaches presented in this paper, perturbations are applied directly after each parametrization. Hence, an interaction of the uncertainty of one physical scheme in the subsequent one is considered in PSPPT and ipSPPT, which seems to increase the stability of the model, but this needs to be confirmed using longer experiments. Of course, sequentially perturbing the partial tendencies implies a possible duplication of model error representation (Christensen et al., 2017). However, the results in Sect. 3 have shown that a significant increase in spread goes along with only a small effect on the model error (RMSE) when applying pSPPT (ipSPPT). A direct comparison of the pSPPT and iSPPT approaches within the C-LAEF framework would be very interesting at this point, but it is beyond the scope of this paper and is planned in a future study. The very flexible structure of the pSPPT approach also allows for combination with other uncertainty representations such as the parameter perturbations scheme in Ollinaho et al. (2017)

The ipSPPT approach is a modification of pSPPT where the tendencies of the variables $T, U, V$, and $Q$ receive separate perturbations. As shown in Sect. 3, this approach obtains the best probabilistic scores overall, even though the method is considered unsatisfactory from a physical point of view. A major concern with the ipSPPT approach is that the balance between the quantities resulting from one parametrization scheme can be disturbed (Palmer et al., 2009). For example, the microphysics scheme can provide an increase in temperature at a certain point due to condensation processes, which are also decreasing the water vapor content. This equilibrium is destroyed if temperature and water vapor content tendencies are perturbed with opposite signs. On the other hand, it seems wrong to assume that $T$ and $Q$ have exactly the same error characteristics, as it is supposed in SPPT and pSPPT. Furthermore, in SPPT and pSPPT the wind direction is never altered stochastically, since the tendencies of the $U$ and $V$ components are always using the same stochastic pattern. Testing over a longer period will be necessary to identify if conservation rules are violated in ipSPPT and if it is really applicable in an operational framework.

Last but not least, perturbations in SPPT are only active in areas where the net tendency is not 0 , even though the individual physical parametrization schemes might have strong opposite contributions. This shortcoming is avoided by perturbing the partial tendencies of the physics parametrizations in both $\mathrm{pSPPT}$ and ipSPPT.

In our experiments no ensemble data assimilation or errors in the initial conditions are taken into account. Consequently, only the impact of different stochastic physics approaches compared to a reference ensemble has been considered. The focus on relative scores between the different experiments also somewhat justifies the fact that we did not consider observation error simulations in our verification. Of course, in- cluding observation error can have a strong impact on scores like ensemble spread (Bouttier et al., 2012), but we suppose that it would act in the same direction for all experiments and therefore the relative conclusions stay the same.

The next step in the development of C-LAEF is to introduce the new stochastic perturbation schemes to a full system with data assimilation and initial perturbations. The verification in this operational framework will show the operational benefit of these new approaches for the C-LAEF system.

Code and data availability. The C-LAEF and AROME codes including all related intellectual property rights, are owned by the members of the LACE and ALADIN consortia. Access to the ALADIN and AROME systems, or elements thereof, can be granted upon request and for research purposes only. INCA data are only available subject to a license agreement with ZAMG (klima@zamg.ac.at).

Author contributions. CW developed the different stochastic schemes together with YW. CW designed the experiments and carried them out together with $\mathrm{CW}$. AA was responsible for the verification of the results. $\mathrm{CW}$ prepared the manuscript with contributions from all co-authors.

Competing interests. The authors declare that they have no conflict of interest.

Acknowledgements. The authors gratefully thank all of the colleagues who contributed to this study. Special thanks go to Eric Bazile and Yann Seity from Météo-France for their input into this work through discussions and to the ECMWF for the possibility to run all the experiments on their supercomputer.

Edited by: Richard Neale

Reviewed by: Michael Denhard and one anonymous referee

\section{References}

Bénard, P., Vivoda, J., Mašek, J., Smolıková, P., Yessad, K., Smith, C., Brožková, R., and Geleyn, J. F.: Dynamical kernel of the Aladin-NH spectral limited-area model: revised formulation and sensitivity experiments, Q. J. Roy. Meteor. Soc., 139, 155-169, https://doi.org/10.1002/qj.522, 2010.

Bengtsson, L., Steinheimer, M., Bechtold, P., and Geleyn, J. F.: A stochastic parametrization for deep convection using cellular automata, Q. J. Roy. Meteor. Soc., 139, 1533-1543, https://doi.org/10.1002/qj.2108, 2013.

Bengtsson, L., Andrae, U., Aspelien, T., Batrak, Y., Calvo, J., de Rooy, W., Gleeson, E., Hansen-Sass, B., Homleid, M., Hortal, M., Ivarsson, K., Lenderink, G., Niemelä, S., Nielsen, K. P., Onvlee, J., Rontu, L., Samuelsson, P., Muñoz, D., S., Subias, A., Tijm, S., Toll, V., Yang, X., and Køltzow, M. Ø.: The HARMONIE - AROME Model Configuration in the ALADIN - 
HIRLAM NWP System, Mon. Weather Rev., 145, 1919-1935, https://doi.org/10.1175/MWR-D-16-0417.1, 2017.

Berner, J., Shutts, G. J., Leutbecher, M., and Palmer, T. N.: A spectral stochastic kinetic energy backscatter scheme and its impact on flow dependent predictability in the ECMWF ensemble prediction system, J. Atmos. Sci., 66, 603-626, https://doi.org/10.1175/2008JAS2677.1, 2009.

Berner, J., Fossell, K. R., Ha, S. Y., Hacker, J. P., and Snyder, C.: Increasing the skill of probabilistic forecasts: Understanding performance improvements from model-error representations, Mon. Weather Rev., 143, 1295-1320, https://doi.org/10.1175/MWRD-14-00091.1, 2015.

Berre, L.: Estimation of synoptic and mesoscale forecast error covariances in a limited area model, Mon. Weather Rev., 128, 644-667, https://doi.org/10.1175/15200493(2000)128<0644:EOSAMF>2.0.CO;2, 2000.

Boisserie, M., Arbogast, P., Descamps, L., Pannekoucke, O., and Raynaud, L.: Estimating and diagnosing model error variances in the Meteo-France global NWP model, Q. J. Roy. Meteor. Soc., 140, 846-854, https://doi.org/10.1002/qj.2173, 2013.

Bouteloup, Y., Seity, Y., and Bazile, E.: Description of the sedimentation scheme used operationally in all Météo-France NWP models, Tellus A, 63, 300-311, https://doi.org/10.1111/j.16000870.2010.00484.x, 2011.

Bouttier, F., Vié, B., Nuissier, O., and Raynaud, L.: Impact of Stochastic Physics in a Convection-Permitting Ensemble, Mon. Weather Rev., 140, 3706-3721, https://doi.org/10.1175/MWRD-12-00031.1, 2012.

Bowler, N. E., Arribas, A., Mylne, K. R., Robertson, K. B., and Beare, S. E.: The MOGREPS short-range ensemble prediction system, Q. J. Roy. Meteor. Soc., 134, 703-722, https://doi.org/10.1002/qj.234, 2008.

Bubnova, R., Hello, G., Bénard, P., and Geleyn, J. F.: Integration of the fully elastic equations cast in the hydrostatic pressure terrain-following coordinate in the framework of the ARPEGE/ALADIN NWP system, Mon. Weather Rev., 123, 515-535, https://doi.org/10.1175/15200493(1995)123<0515:IOTFEE>2.0.CO;2, 1995.

Buizza, R., Miller, M., and Palmer, T. N.: Stochastic representation of model uncertainties in the ECMWF ensemble prediction system, Q. J. Roy. Meteor. Soc., 125, 2887-2908, 1999.

Christensen, H. M., Lock, S.-J., Moroz, I. M., and Palmer, T. N.: Introducing independent patterns into the Stochastically Perturbed Parametrization Tendencies (SPPT) scheme, Q. J. Roy. Meteor. Soc., 143, 2168-2181, https://doi.org/10.1002/qj.3075, 2017.

Cuxart, J., Bougeault, P., and Redelsperger, J.-L.: A turbulence scheme allowing for mesoscale and largeeddy simulations, Q. J. Roy. Meteor. Soc., 126, 1-30, https://doi.org/10.1002/qj.49712656202, 2000.

Davies, H.: A lateral boundary formulation for multi-level prediction models, Q. J. Roy. Meteor. Soc., 102, 405-418, https://doi.org/10.1002/qj.49710243210, 1976.

Davini, P., von Hardenberg, J., Corti, S., Christensen, H. M., Juricke, S., Subramanian, A., Watson, P. A. G., Weisheimer, A., and Palmer, T. N.: Climate SPHINX: evaluating the impact of resolution and stochastic physics parametrisations in the ECEarth global climate model, Geosci. Model Dev., 10, 1383-1402, https://doi.org/10.5194/gmd-10-1383-2017, 2017.
Fouquart, Y. and Bonnel, B.: Computations of solar heating of the earth's atmosphere: A new parameterization, Beitr. Phys. Atmos., 53, 35-62, https://doi.org/10.1029/JD093iD09p11063, 1980.

Haiden, T., Kann, A., Wittmann, C., Pistotnik, G., Bica, B., and Gruber, C.: The Integrated Nowcasting through Comprehensive Analysis (INCA) System and Its Validation over the Eastern Alpine Region, Weather Forecast., 26, 166-183, https://doi.org/10.1175/2010WAF2222451.1, 2011.

Hamill, T. and Colucci, S. J.: Verification of EtaRSM short-range ensemble forecasts, Mon. Weather Rev., 125, 1312-1327, https://doi.org/10.1175/15200493(1997)125<1312:VOERSR>2.0.CO;2, 1997.

Isaksen, L., Bonavita, M., Buizza, R., Fisher, M., Haseler, J., Leutbecher, M., and Raynaud, L.: Ensemble of data assimilations at ECMWF, Tech. Mem. ECMWF, 636, 1-48, 2010.

Kober, K. and Craig, G. C.: Physically based stochastic perturbations (PSP) in the boundary layer to represent uncertainty in convective initiation, J. Atmos. Sci., 73, 2893-2911, https://doi.org/10.1175/JAS-D-15-0144.1, 2016.

Leutbecher, M. and Lang, S. T. K.: On the reliability of ensemble variance in subspaces defined by singular vectors, Q. J. Roy. Meteor. Soc., 140, 1453-1466, https://doi.org/10.1002/qj.2229, 2013.

Leutbecher, M., Lock, S., Ollinaho, P., Lang, S. T., Balsamo, G., Bechtold, P. , Bonavita, M., Christensen, H. M., Diamantakis, M., Dutra, E., English, S., Fisher, M., Forbes, R. M., Goddard, J., Haiden, T., Hogan, R. J., Juricke, S., Lawrence, H., MacLeod, D., Magnusson, L., Malardel, S., Massart, S., Sandu, I., Smolarkiewicz, P. K., Subramanian, A., Vitart, F., Wedi, N., and Weisheimer, A.: Stochastic representations of model uncertainties at ECMWF: State of the art and future vision, Q. J. Roy. Meteor. Soc., 143, 2315-2339, https://doi.org/10.1002/qj.3094, 2017.

Mascart, P. J. and Bougeault, P.: The Meso-NH atmospheric simulation system: Scientific documentation, Tech. rep. Meteo France, 2011.

Masson, V., Le Moigne, P., Martin, E., Faroux, S., Alias, A., Alkama, R., Belamari, S., Barbu, A., Boone, A., Bouyssel, F., Brousseau, P., Brun, E., Calvet, J.-C., Carrer, D., Decharme, B., Delire, C., Donier, S., Essaouini, K., Gibelin, A.-L., Giordani, H., Habets, F., Jidane, M., Kerdraon, G., Kourzeneva, E., Lafaysse, M., Lafont, S., Lebeaupin Brossier, C., Lemonsu, A., Mahfouf, J.-F., Marguinaud, P., Mokhtari, M., Morin, S., Pigeon, G., Salgado, R., Seity, Y., Taillefer, F., Tanguy, G., Tulet, P., Vincendon, B., Vionnet, V., and Voldoire, A.: The SURFEXv7.2 land and ocean surface platform for coupled or offline simulation of earth surface variables and fluxes, Geosci. Model. Dev., 6, 929-960, https://doi.org/10.5194/gmd-6-929-2013, 2013.

Mlawer, E. J., Taubman, S. J., Brown, P. D., Iacono, M. J., and Clough, S. A.: Radiative transfer for inhomogeneous atmospheres: RRTM, a validated correlated-k model for the longwave, J. Geophys. Res.-Atmos., 102, 663-682, https://doi.org/10.1029/97JD00237, 1997.

Ollinaho, P., Lock, S.-J., Leutbecher, M., Bechtold, P., Beljaars, A., Bozzo, A., Forbes, R. M., Haiden, T., Hogan, R., and Sandu, I.: Towards process-level representation of model uncertainties: Stochastically perturbed parametrisations in the 
ECMWF ensemble, Q. J. Roy. Meteor. Soc., 143, 408-422, https://doi.org/10.1002/qj.2931, 2017.

Palmer, T. N., Buizza, R., Doblas-Reyes, F., Jung, T., Leutbecher, M., Shutts, G. J., Steinheimer, M., and Weisheimer, A.: Stochastic parametrization and model uncertainty, Tech. Mem. ECMWF, 598, available at: https://www.ecmwf.int/en/elibrary/ 11577-stochastic-parametrization-and-model-uncertainty (last access: 10 July 2018), 2009.

Pergaud, J., Masson, V., and Malardel, S.: A parameterization of dry thermals and shallow cumuli for mesoscale numerical weather prediction, Bound.-Layer Meteor., 132, 83-106, https://doi.org/10.1007/s10546-009-9388-0, 2009.

Pinty, J.-P. and Jabouille, P.: A mixed-phase cloud parameterization for use in mesoscale non-hydrostatic model: simulations of a squall line and of orographic precipitations, Proc. Conf. Cloud Phys., 1999, 217-220, https://doi.org/10.1256/qj.02.50, 1998.

Plant, R. S. and Craig, G. C.: A stochastic parameterization for deep convection based on equilibrium statistics, J. Atmos. Sci., 65, 87-104, https://doi.org/10.1175/2007JAS2263.1, 2008.

Sanchez, C., Williams, K. D., and Collins, M.: Improved stochastic physics schemes for global weather and climate models, Q. J. Roy. Meteor. Soc., 142, 147-159, https://doi.org/10.1002/qj.2640, 2016.

Seity, Y., Brousseau, P., Malardel, S., Hello, G., Bénard, P., Bouttier, F., Lac, C., and Masson, V.: The AROME-France ConvectiveScale Operational Model, Mon. Weather Rev., 139, 976-991, https://doi.org/10.1175/2010MWR3425.1, 2011.

Shutts, G. J. and Pallares, A. C.: Assessing parametrization uncertainty associated with horizontal resolution in numerical weather prediction models, Philos. Trans. R. Soc. A, 372, 1-14, 2014.

Szúcs, M.: SPPT in AROME and ALARO, Presentation at HIRLAM WW on EPS and Predictability, available at: http://www.rclace.eu/File/Predictability/2014/LACE_report_ Mihaly_Szucs_2014.pdf (last access: 15 July 2018), 2016.
Termonia, P., Fischer, C., Bazile, E., Bouyssel, F., Brožková, R., Bénard, P., Bochenek, B., Degrauwe, D., Derková, M., El Khatib, R., Hamdi, R., Mašek, J., Pottier, P., Pristov, N., Seity, Y., Smolíková, P., Španiel, O., Tudor, M., Wang, Y., Wittmann, C., and Joly, A.: The ALADIN System and its Canonical Model Configurations AROME CY41T1 and ALARO CY40T1, Geosci. Model. Dev., 11, 257-281, https://doi.org/10.5194/gmd11-257-2018, 2018

Wang, Y., Meirold-Mautner, I., Kann, A., Ŝajn Slak, A., Simon, A., Vivoda, J., Bica, B., Böcskör, E., Brezková, L., Dantinger, J., Giszterowicz, M., Heizler, G., Iwanski, R., Jachs, S., Bernard, T., Kršmanc, R., Merše, J., Micheletti, S., Schmid, F., Steininger, M., Haiden, T., Regec, A., Buzzi, M., Derková, M., Kozarić, T., Qiu, X., Reyniers, M., Yang, J., Huang, Y., and Vadislavsky, E.: Integrating nowcasting with crisis management and risk prevention in a transnational and interdisciplinary framework, Meteor. Z., 26, 459-473, https://doi.org/10.1127/metz/2017/0843, 2017.

Wang, Y., Belluš, M., Ehrlich, A., Mile, M., Pristov, N., Smoliková, P., Španiel, O., Trojáková, A., Brožkov, R., Cedelnik, J., Klarić, D., Kovačić, T., Mašek, J., Meier, F., Szintai, B., Tascu, S., Vivoda, Wastl, C., and Wittmann, C.: 27 years of Regional Cooperation for Limited Area Modelling in Central Europe (RC LACE), B. Am. Meteorol. Soc., 99, 1415-1432, https://doi.org/10.1175/BAMS-D-16-0321.1, 2018.

Weidle, F., Wang, Y., Tian, W., and Wang, T.: Validation of strategies using Clustering analysis of ECMWFEPS for initial perturbations in a Limited Area Model Ensemble Prediction System, Atmos.-Ocean, 51, 248-295, https://doi.org/10.1080/07055900.2013.802217, 2013.

Weisheimer, A., Corti, S., Palmer, T. N., and Vitart, F.: Addressing model error through atmospheric stochastic physical parametrizations: impact on the coupled ECMWF seasonal forecasting system, Philos. Trans. R. Soc. A, 372, 1-21, https://doi.org/10.1098/rsta.2013.0284, 2014.

Wilks, D.: Statistical Methods in the Atmospheric Sciences, Volume 100, 3rd Edition, Academic Press, 704 pp., 2011. 